\title{
PHASE TRANSITIONS, DOMAIN WALLS, AND DARK MATTER
}

\author{
W-Y. P. HWANG \\ Institute of Astrophysics \& Department of Physics, \\ National Taiwan University, Taipei, Taiwan, R.O.C. \\ wyhwang@phys.ntu.edu.tw
}

\begin{abstract}
We discuss possible roles in the Early Universe of the electroweak (EW) phase transition, which endows masses to the various particles, and the QCD phase transition, which gives rise to quark confinement and chiral symmetry breaking. Both phase transitions are well-established phenomena in the standard model of particle physics. Presumably, the EW phase transition would have taken place in the early universe at around $10^{-11} \mathrm{sec}$, or at the temperature of about $300 \mathrm{GeV}$ while QCD phase transition occurred between $10^{-5} \mathrm{sec}$ and $10^{-4} \mathrm{sec}$, or at about $150 \mathrm{MeV}$. In this article, I wish to model the EW or QCD phase transition in the early universe as driven by a complex scalar field with spontaneous symmetry breaking such that the continuous degeneracy of the true ground states can be well represented. Specific interest has been directed to nucleation of domains, production of domain walls, and subsequent re-organization of domain walls resulting in "domain-wall nuggets". It is suggested that the domain-wall nuggets contribute to dark matter in the present Universe.
\end{abstract}

Keywords: Early Universe; phase transitions; domain-wall nuggets.

PACS Nos.: 12.15.Ji, 12.38.Lg

\section{Introduction}

The discovery ${ }^{1}$ of fluctuations or anisotropies, at the level of $10^{-5}$, associated with the cosmic microwave background (CMB) has helped transformed the physics of the early universe into a main-stream research area in astronomy and in particle astrophysics, both theoretically and observationally ${ }^{2}$. CMB anisotropies and polarizations, the latter beginning to be observed recently ${ }^{3,4}$, are linked closely to the inhomogeneities produced in the early Universe. Such inhomogeneities are often attributed to quantum fluctuations produced during the early inflationary stage, as might be amplified many orders of magnitude by the inflation. In this article, we wish to examine possible roles of phase transitions, an inevitable process in the hot big bang era, in amplifying the existing inhomogeneities or generating inhomogeneities of new kinds.

Over the last three decades, the standard model of particle physics has been well established to the precision level of $10^{-5}$ or better in the electroweak sector. In the theory, the electroweak (EW) phase transition, which endows masses to the various particles, and the QCD phase transition, which gives rise to confinement of quarks 
and gluons within hadrons in the true QCD vacuum, are two well-established phenomena. Presumably, the EW and QCD phase transitions would have taken place in the early universe, respectively, at around $10^{-11} \mathrm{sec}$ and at a time between $10^{-5} \mathrm{sec}$ and $10^{-4} \mathrm{sec}$, or at the temperature of about $300 \mathrm{GeV}$ and of about $150 \mathrm{MeV}$, respectively. Indeed, it has become imperative to formulate the EW and QCD phase transitions in the early universe if a quantitative theory of cosmology can ever be obtained.

\section{Phase Transitions in the Early Universe}

When the Universe expands, it cools, from an initially very high temperature built up from the reheating at the end of the inflation era eventually down to the critical temperature $T_{c}$ for the electroweak phase transition. Just above $T_{c}$, say at $T=$ $T_{c}+\delta$ with $\delta$ a small fraction of $T_{c}$, bubbles of stable vacuum begin to nucleate at spots with temperature lower than $T_{c}$ or at spots at higher density - due to the inhomogeneities already created during the inflation era. To describe the phase transition phenomenologically, we use the complex scalar field with a spontaneoussymmetry-breaking potential:

$$
V(\phi)=\mu^{2}(T) \phi^{*} \phi+\lambda(T)\left(\phi^{*} \phi\right)^{2}
$$

with $\mu^{2}(T)<0$ for $T<T_{c}$ and $\lambda(T)$ always positive (with its temperature dependence neglected henceforth). We note that the lower-temperature phase of the EW or QCD phase transitions is naturally degenerate, such as $\theta$-vacua in QCD or in some other degree(s) in the EW phase transition, such that the description presented here may be of general applicability.

The temperature dependence of $\mu^{(T)}$ near $T_{c}$ is an issue of critical importance and more investigation is needed. It could happen that $\mu^{2}(T)$ is just a step function across the critical temperature, signaling a "strong" first-order phase transition. It could also happen that $\mu^{2}(T)$ is a smooth function of $T$ and crosses zero becoming negative when $T$ cools and crosses $T_{c}$. But $\mu^{2}(T)$ should have stabilized at some negative constant at a temperature not too far below $T_{c}$. In the present situation, it is difficult to determine the order of the phase transition - it could still be first-order but second-order or even higher becomes possible.

For the sake of simplicity and also as our first attempt, we assume that $\mu^{2}(T)$ is a step function - in the Wigner phase above $T_{c}$ while in the Nambu-Goldstone phase below $T_{c}$. In this case, the number of bubbles nucleated early on when $T$ is still slightly above $T_{c}$ is expected to be negligibly smaller than the number of those bubbles nucleated when the universe cools to already cross $T_{c}$, but presumably above the so-called "supercooling" temperature $T_{s}$ when the entire system should have converted itself to the lower-temperature phase, i.e., the true vacuum. Nevertheless, the initial inhomogeneities, however tiny, would determine the various locations at which the bubbles first appear and the subsequent natural bubble expansion serves to amplify the amplitudes of these inhomogeneities. 
Our strategy of treating the problem of first-order phase transition is to first subdivide it into four steps with different questions, viz.: (1) how a bubble of true vacuum grows or shrinks in a false vacuum; (2) how two growing bubbles collide and merge with each other, separating the domains by "domain walls"; (3) how the bubbles of various sizes nucleate or grow out of the false vacuum as the temperature lowers (due to the expansion of the universe), or how to categorize such statistical ensemble of bubbles; and (4) how specific objects, such as domain walls, vortex strings, and even black holes, get produced during the specific phase transition. In this article, I shall not enter much of the details regarding numerical solutions but will try to describe in clarity what one can do on the problem of first-order phase transitions in the early universe.

We probably should provide another short review on the hot big bang history of our Universe. Recall that the prevailing view regarding our universe, or the $3+1$ dimensional space-time, is that it originates from the joint making of Einstein's general relativity and the cosmological principle while the observed anisotropies may stem from quantum fluctuations in the inflation era. The cosmological principle state that our universe is homogeneous and isotropic, leading to the Robertson-Walker metric ${ }^{5}$.

$$
d s^{2}=d t^{2}-R^{2}(t)\left\{\frac{d r^{2}}{1-k r^{2}}+r^{2} d \theta^{2}+r^{2} \sin ^{2} \theta d \phi^{2}\right\}
$$

Here the parameter $k$ describes the spatial curvature with $k=+1,-1$, and 0 referring to an open, closed, and flat universe, respectively. The scale factor $R(t)$ describes the size of the universe at time $t$.

To a reasonable first approximation, the universe can be described by a perfect fluid, i.e., a fluid with the energy-momentum tensor $T_{\nu}^{\mu}=\operatorname{diag}(\rho,,-p,-p,-p)$ where $\rho$ is the energy density and $p$ the pressure. Thus, the Einstein equation, $G_{\nu}^{\mu}=8 \pi G_{N} T_{\nu}^{\mu}+\Lambda g^{\mu}{ }_{\nu}$, gives rise to only two independent equations, i.e., from $(\mu, \nu)=(0,0)$ and $(i, i)$ components,

$$
\begin{gathered}
\frac{\dot{R}^{2}}{R^{2}}+\frac{k}{R^{2}}=\frac{8 \pi G_{N}}{3} \rho+\frac{\Lambda}{3} . \\
2 \frac{\ddot{R}}{R}+\frac{\dot{R}^{2}}{R^{2}}+\frac{k}{R^{2}}=-8 \pi G_{N} p+\Lambda .
\end{gathered}
$$

Combining with the equation of state (EOS), i.e. the relation between the pressure $p$ and the energy density $\rho$, we can solve the three functions $R(t), \rho(t)$, and $p(t)$ from the three equations. Further, the above two equations yields

$$
\frac{\ddot{R}}{R}=-\frac{4 \pi G_{N}}{3}(\rho+3 p)+\frac{\Lambda}{3},
$$

showing either that there is a positive cosmological constant or that $\rho+3 p$ must be somehow negative, since the expansion of our present universe is still accelerating $\left(\frac{\ddot{R}}{R}>0\right)$, as from Supernovae Type Ia observations ${ }^{6}$ as well as from WMAP CMB measurements. The "matter" or energy form with negative $\rho+3 p$ has been referred 
to as "quintessence" 7 by Paul Steinhardt] while that responsible for the acceleration of our Universe's expansion is called "dark energy" [by Mike Turner].

Using these equations, we have $\rho \propto R^{-4}$ for a radiation-dominated universe $(p=$ $\rho / 3)$ while $\rho \propto R^{-3}$ for a matter-dominated universe $(p<<\rho)$. CMB observations indicate that our universe is flat, i.e., its energy density being the critical density $\rho_{c}=8.0 \times 10^{-30} \mathrm{~g} / \mathrm{cm}^{3}$. The CMB observations ${ }^{8}$, coupled with other large-scale observations, already fix the energy budget of our present Universe - $70 \%$ in the form of "dark energy" (which is responsible for the accelerating status of the present Universe), about $25 \%$ in "dark matter", and only slightly less than $5 \%$ being baryons (and only $10 \%$ of the baryon content making up all the galaxies).

\section{Bubble Nucleation and Expansion in the False Vacuum}

Consider a spherical wall of radius $R$ and thickness $\Delta$ separating the true vacuum inside from the false vacuum outside. The energy density difference of the vacua is $B$, the bag constant in the most simplified situation, and the energy $\sigma$ per unit area associated with the surface tension on the separating wall is a quantity to be calculated but nevertheless is small compared to the latent heat. If the wall expands outward for a distance $\delta R$, then the energy budget, in its first approximation, arising from the bubble change is

$$
\frac{\mu^{4}}{\lambda} \cdot 4 \pi R^{2} \cdot \delta R-\sigma 4 \pi\left\{(R+\delta R)^{2}-R^{2}\right\}=\gamma \sigma 4 \pi R^{2} \dot{v} \delta R .
$$

With $\delta R=\dot{R} \delta t$ and $x \equiv R(t)$, we obtain

$$
\frac{\mu^{4}}{\lambda}-\frac{2 \sigma}{x}=\gamma \sigma \ddot{x}
$$

The equation can be solved, showing the existence of a "critical" radius beyond which the bubble can continue to expand, or otherwise shrinks.

How can we obtain solutions to the bubble in the false vacuum? We return to the complex scalar potential given earlier. The critical point which we wish to make is that if we assume that the dynamics of bubbles is dictated by the such scalar dynamics then the various questions regarding bubble nucleation, bubble merging, and other subsequent bubble dynamics can be answered without ambiguity. One major issue has to do with the fact that, in the complex scalar field description, the true vacua have degeneracy described by a continuous real parameter $\theta$. Although the Higgs mechanism in the minimal electroweak theory has eaten up such degeneracy, it is nevertheless a common expectation that other degrees of freedom such as gauge fields and matter fields, or aspects beyond the minimal standard framework, bring in continuous degeneracy of the true vacua. For the sake of simplicity, we may use the phase of the complex scalar field to label the continuous degeneracy of the true vacua.

We note that the false vacuum is described by $\phi=0$ everywhere in the spacetime. Consider the solution for a bubble of true vacuum in this environment. It is required 
that the field $\phi$ must satisfy the field equation everywhere in spacetime, including crossing the wall of thickness $\Delta$ to connect smoothly the true vacuum inside and the false vacuum outside - this in fact results in a constant $\theta$ across the thin surface. This is why we may call the bubble solution "a soliton", in the sense of a nontopological soliton of T.D. Lee's. However, the soliton may grow in an accelerating way, or thus the name "exploding soliton".

The situation must have changed so much that at a very short instant later the universe expands even further and cools to even a little more farther away from $T_{c}$ and most places in the universe must be in the true vacuum, making the previously false vacuum shrink and fractured into small regions of false vacua, presumably dominantly in spherical shape, which is shrinking in an accelerating way, or "implosively". Using again the complex scalar field as our language, we then have "imploding solitons".

The question of an "exploding" or "imploding" soliton solution can be solved for the complex scalar field as stated above - an exercise which we shall not go into any detail here. A $\phi$ field everywhere in the space-time can be found as the solution to the field equations. The surface tension can be calculated.

\section{Dynamics of Domain Walls}

When bubbles of true vacua grow explosively, the nearby pair of bubbles will soon squeeze or "collide with" each other, resulting in formation of a "domain wall" between the two bubbles (of two different $\theta$ 's) while producing objects that have specific coupling to the system. The situation is again complicated. Nevetheless, we may try to disentangle the complexities by looking at between the two bubble walls that are almost ready to touch and for the initial attempt neglecting the coupling of the vacuum dynamics to the matter content. Between the two bubble walls, especially between the centers of the two bubbles, it looks like a problem of plane walls in collision - and this is where we try to solve the problem to begin with.

Consider the problem of two walls approaching each other. The wall, each of thickness $\Delta$, separates the true vacuum on one side from the false vacuum on the other side of the wall. For the sake of simplicity, both walls may be assumed parallel to the $(x y)$-plane and are infinite in both the $x$ and $y$ directions. In addition, the wall at $z=R$ is moving to the left with the velocity $v$ while that at $z=-R$ moving to the right with $+v$. For $z>R+\frac{\Delta}{2}$ and all $x$ and $y$, the complex scalar field $\phi$ assumes $\phi_{0}$, a value of the true vacuum (the ground state). On the other hand, for $z<-R-\frac{\Delta}{2}$ and all $x$ and $y$, the complex scalar field $\phi$ assumes $\phi_{0}^{\prime}$, another value of the true vacuum which differs in general from $\phi_{0}$. In between the two walls, i.e., $-R+\frac{\delta}{2}<z<R-\frac{\Delta}{2}$, we have $\phi=0$, the false vacuum. As indicated earlier, the field $\phi$ must satisfy the field equation everywhere in spacetime:

$$
\frac{\partial^{2} \phi}{\partial z^{2}}-\frac{\partial^{2} \phi}{\partial t^{2}}=V^{\prime}(\phi) .
$$

We may write the wall on the right hand side but moving toward the left with 
the velocity $v$ :

$$
\phi=f(z-v t), \quad \text { for } z-v t>0, t<R / v .
$$

so that

$$
\left(1-v^{2}\right) f^{\prime \prime}=\lambda f\left(|f|^{2}-\sigma^{2}\right), \quad \sigma \equiv\left|\phi_{0}\right|>0 .
$$

We may write the complex scalar function $f$ as follows:

$$
f \equiv u e^{i \theta}
$$

so that, with $\tilde{\lambda} \equiv \lambda /\left(1-v^{2}\right)$,

$$
\begin{gathered}
u^{\prime \prime}-u\left(\theta^{\prime}\right)^{2}=\tilde{\lambda} u\left(u^{2}-\sigma^{2}\right), \\
2 u^{\prime} \theta^{\prime}+u \theta^{\prime \prime}=0 .
\end{gathered}
$$

Integrating the second equation, we find

$$
u^{2} \theta^{\prime}=J,
$$

with $J$ an integation constant. The $u$-equation can now be integrated:

$$
\left(u^{\prime}\right)^{2}=-\frac{J^{2}}{u^{2}}+\tilde{\lambda}\left(\frac{u^{4}}{2}-\sigma^{2} u^{2}+\frac{K}{2}\right),
$$

with $K$ another integration constant.

Accordingly, in connecting a true vacuum (with $u=\sigma$ ) with a false vacuum (with $u=0$ ), we must choose $J=0$ across the thin layer separating the two regions, in order to avoid the potential singularity near $u=0$. In this case, we obtain

$$
\xi \propto \pm \int_{0}^{\sigma} \frac{d u}{\sqrt{\left(u^{2}-\sigma^{2}\right)^{2}-\sigma^{4}+K}}
$$

an equation determining how $u$ varies with $\xi$.

The situation for connecting the two regions with true vacua does not require $J=0$ - the integration over $\theta^{\prime}$ should give the difference in the $\theta$ values while the $\frac{d u}{d \xi}$ starts off with some negative value, varies to zero in the middle, and changes to positive values (by choosing another sign in the last equation. This is the "domainwall" solution.

This is not the end of the story. At the completion of the phase transition, we have the situation as described below:

- Domain walls separate the true vacua of different $\theta_{i}$.

- Vacua and walls fill up the entire space-time, a topological honeycomb-like solution to the field equations for $\phi(x)$.

- Domain walls can expand or shrink but, unless there is an extra coupling or interaction effective at a similar time scale set by $\mu^{2}$ and $\lambda$, cannot decay and thus cannot disappear.

- The "line" or tiny "tube" separating the three different vacua can be in the form of a vortex or a string with a false vacuum in the middle. 
Consequently, some bubbles continue to grow at the expense of shrinking many others. Just like cluster simulations of $N$ mass points, the nodes tend to cluster or clump, with the time scale set by $\mu^{2}$ and $\lambda$ - forming the "domain-wall nuggets" shortly after the completion of phase transitions. By calculating the total energy associated with the domain walls and the nuggets, we realize that such domain-wall nuggets could well contribute to the "dark matter" component of the present-day Universe.

\section{Discussions}

Phase transitions occurred in the hot big bang era of the early Universe, making the already delicate situation even more sophisticated. Bubbles of true vacua nucleate in the false vacuum. The field equations can be solved in the connecting thin layer, leading to expansion or shrinkage of the originally small bubble. The two truevacuum bubbles grow, collide, or merge by forming a domain wall between the two regions. When the entire universe turns over with true vacua everywhere, between each pair separated by domain walls. The system will continue to evolve - some domains grow at the expense of shrinking many others resulting in "domain-wall nuggets".

Unless some new dynamics is introduced, domain walls or nuggets cannot disappear altogether. This is true so long as we insist that everything follows from the field equations for the complex scalar fields. So long as domain-wall nuggets cannot decay in short times, they might contribute to inhomogeneities, as reflected in the CMB observation of the last-scattering surface (LSS), when the Universe was about 300,000 years old - such inhomogeneities can at most be at the level of one part in 100,000 . Similar words can be said regarding black holes or other topological objects (such as vortex strings). In any event, the role of phase transitions in producing topological defects as inhomogeneities of new kinds or amplifying the existing tiny inhomogeneities produced at the earlier epoch(s) need to be investigated in greater details and in broader context.

There are important questions which are of critical importance and yet require in-depth studies, viz.: What is the temperature dependence of $\left.\mu^{(} T\right)$ ? How can we determine in a decisive manner the order of the EW and/or QCD phase transition? Is the continuous degeneracy of the true vacua in the $\mathrm{EW}$ phase transition genuinely there, like the $\theta$-vacua in the QCD phase transition? What is the supercooling temperature and how is it calculated? With so many unsolved important questions in mind, we would have to close our discussions with many question marks!!

\section{Acknowledgments}

This work is supported primarily through the Taiwan CosPA Project, as funded by the Ministry of Education (89-N-FA01-1-0 up to 89-N-FA01-1-5). It is also supported in part by National Science Council of R.O.C. (NSC 91-2112-M002-041 \& NSC 922112-M002-051). 


\section{References}

1. G. Smoot et al., Astrophys. J. 396 (1992) L1; C. Bennett et al., Astrophys. J. 396 (1992) L7; E. Wright et aql., Astrophys. J. 396 (1992) L11.

2. C. L. Bennett, M. S. Turner, and M. White, Physics Today, November 1997, p. 32, for an early general review.

3. E.M. Leitch et al., arXiv:astro-ph/0209476, September 2002; J. Kovac et al., arXiv:astro-ph/0209478, September 2002.

4. A. Kogut et al., arXiv:astro-ph/0302213, February 2003.

5. E.W. Kolb and M.S. Turner, The Early universe (1994) Addison-Wesley Publishing Co., New York.

6. S. Perlmutter et al. [Supernova Cosmology Project], Astrophys. J. 517 (1999) 565; A. G. Riess et al. [Supernova Search Team], Astron. J. 116(1998) 1009.

7. R.R. Caldwell, R. Dave, and P.J. Steinhardt, Phys. Rev. Lett. 80, 1582 (1998).

8. D.N. Spergel et al., "First Year Wilkinson Microwave Anisotropy Probe Observations: Determination of Cosmological Parameters", February 2003. 